\title{
Efficacy of 6\% Sodium Hypochlorite and 2\% Glutaraldehyde in Decontamination Of Surgical Instruments. an in-Use Assay
}

\author{
R. Akshaya ${ }^{1}$ and N.P. Muralidharan ${ }^{2}$ \\ ${ }^{1}$ Undergraduate Student Saveetha Dental College, Saveetha Institute of Medical and \\ Technical Sciences, Saveetha University, Chennai-600077, Tamil Nadu, India. \\ ${ }^{2}$ Associate professor, Department of microbiology, Saveetha Dental College, Saveetha Institute \\ of Medical and Technical Sciences Saveetha University, Chennai-600077, Tamil Nadu, India
}

\section{ABSTRACT}

Soiled surgical instruments are always carrying the risk of transmitting the infection while handling and cleaning. As per the CDC and ADA recommendation, decontamination is essential to avoid the risk of transmission of blood borne pathogens. Two chemicals are universally accepted for this purpose, 6\% sodium hypochlorite and 2\% glutaraldehyde. This study is done to find out the efficacy of these two disinfectants in decontaminating the blood stained instruments. Two sets of samples are collected. One set from the discarding jar that contains 6\% sodium hypochlorite and the other set from the jar containing $2 \%$ glutaraldehyde. 10 samples from hypo were collected and 3 samples from the glutaraldehyde are collected so far. The samples were transported to the microbiology laboratory and processed. Exposure time for hypo is 30 mins and for glutaraldehyde it is 2 hours (CDC recommendation). The collected samples were processed without any time delay. $20 \mu \mathrm{l}$ of the sample was transferred to $\mathrm{BHI}$ agar and incubated for $24 \mathrm{hrs}$ at $37^{\circ} \mathrm{C}$ aerobically. After the incubation, the total CFU were calculated and tabulated. It is concluded that $2 \%$ glutaraldehyde shows best results compared to that of 6\% sodium hypochlorite solution in decontamination of soiled surgical instruments. Though both the chemical solutions are universally accepted for decontamination of soiled surgical instruments. The aim of study is to evaluate the effect of decontamination of surgical instruments with $6 \%$ sodium hypochlorite in comparison with $2 \%$ glutaraldehyde.

KEY WORDS: SODIUM HYPOCHLORITE, GLUTARALDEHYDE, SURGICAL INSTRUMENTS, DECONTAMINATION, DISINFECTANTS.

\section{INTRODUCTION}

Sodium hypochlorite (NaOCI) is the most widely used disinfectant in the medical field despite the increasing availability of other disinfectants (Mekonnen et al., 2015).

\section{ARTICLE INFORMATION}

*Corresponding Author: muralidharan@saveetha.com Received 7th Aug 2020 Accepted after revision 25th Sep 2020 Print ISSN: 0974-6455 Online ISSN: 2321-4007 CODEN: BBRCBA

Thomson Reuters ISI Web of Science Clarivate Analytics USA and Crossref Indexed Journal

\section{Clarivate
Analytics}

NAAS Journal Score 2020 (4.31) SJIF: 2020 (7.728)

A Society of Science and Nature Publication,

Bhopal India 2020. All rights reserved.

Online Contents Available at: http//www.bbrc.in/

Doi: http://dx.doi.org/10.21786/bbrc/13.8/124
Sodium hypochlorite is considered as the ideal disinfectant and it has an excellent cleaning action. It is considered to be the effective disinfectant on surgical instruments (Fukuzaki, 2006). Sodium hypochlorite disinfection processes majorly depend on the concentration of the chlorine and the $\mathrm{pH}$ of the solution (Vianna et al., 2004). Despite being one of the most traditional disinfectants, hypochlorite remains studied and compared with other technologies and products. It was presented that hypochlorite showed superior action or equivalence to most other products with broad microbicidal action, including spores, and progressive action as longer exposure time and concentration, especially those related 
to HAIs transmission (Health care associated Infections) (Pereira et al., 2015).

There are various agents for sterilizing dental instruments like dry heat, steam, chemicals etc. They are also widely used for decontamination of soiled surgical instruments (Rani and Others, 2016). Safe and effective decontamination procedures must be carried out before instruments are put into the appropriate equipment for sterilization. These procedures should be performed to remove gross contamination by blood, saliva and dental materials that harbor microorganisms and impair the sterilization process. In addition, the method of handling, concentrations of blood packaging and wrapping instruments during decontamination and sterilization also has become significant due to recent concerns regarding the transmission of blood borne disease (Sanchez and Macdonald, 1995; Ashwin and Muralidharan, 2015). The use of 2\% glutaraldehyde in an automated machine system to decontaminate endoscopes is well established disinfection. This is considered universally for decontamination of instruments and for sterilization (Corcoran, Holton and Ridgway, 1994). Compared to hypo solution , $2 \%$ glutaraldehyde is showing better activity in decontaminating surgical instruments according to this study.

Soiled surgical instruments are always carrying the risk of transmitting the infection while handling and cleaning. As per the CDC and ADA recommendation, decontamination is essential to avoid the risk of transmission of blood borne pathogens (Cardoso et al., 1999). Previous studies have indicated that alkaline $2 \%$ glutaraldehyde was considered as an adequate sterilizing medium for medical and surgical instruments. Two chemicals are universally accepted for this purpose, $6 \%$ sodium hypochlorite and 2\% glutaraldehyde. Sodium Hypochlorite has some antimicrobial activity in endodontic practice in decontamination especially 6\% Sodium hypochlorite as recommended by ADA. It has resistant bacterial spores. In dentistry, sterilization of gutta percha is done with 0.5 to $4 \%$ hypo (Ozalp, Okte and Ozcelik, 2006; Renuka and Muralidharan, 2017). This study has been done to find out the efficacy of these two disinfectants in decontaminating the blood stained instruments.

According to some research, 2\% glutaraldehyde is not capable of disinfecting gutta percha even after 15 mins. It should be kept for 8 to 10 hours for disinfection. Also a low percentage of $\mathrm{NaOCl}$ is effective in decontaminating gutta percha. High percentage may lead to damage in periapical tissues (Paiva et al., 2013; Shahana and Muralidharan, 2016). Instruments Scientific articles and increased publicity about the potential for transmitting infectious agents in dentistry have focused attention on dental instruments as possible agents for pathogen transmission. According to the research by American Dental Association, surgical and other medical instruments which penetrate soft tissues or bones are classified as critical devices that should be sterilized after each use or simply discarded.
Some instruments are not intended to penetrate soft tissues or bone in the oral cavity (e.g., amalgam condensers, and air/water syringes) but that could contact oral tissues are classified as semicritical, but sterilization after each use is recommended if the instruments are heat-tolerant (Rutala and Weber, 2008). The basis of antimicrobial activity, Asporin (2\% alkaline glutaraldehyde) should be recommended for chemical sterilization or high level disinfection of dental instruments and if only disinfection is required, Virkon may be a possible alternative, even if used with a higher exposure time (Angelillo et al., 1998).

In this study, we compared the efficiency of decontamination of surgical instruments with two solutions 6\% Sodium hypochlorite and 2\% Glutaraldehyde. It is tested against bacterial colonies and counted accordingly. Some research studies have concluded that $2 \%$ alkaline glutaraldehyde shows more efficacy in decontamination. These solutions have been considered universally by CDC and ADA.

\section{MATERIAL AND METHODS}

Two sets of samples are collected. One set from the discarding jar that contains 6\% sodium hypochlorite and the other set from the jar containing 2\% glutaraldehyde. 10 samples from hypo were collected and 10 samples from the glutaraldehyde are collected. The samples were transported to the microbiology laboratory and processed. Exposure time for hypo is 30 mins and for glutaraldehyde it is 2 hours (ADA recommendation). The collected samples were processed without any time delay. $20 \mu \mathrm{l}$ of the sample was transferred to BHI agar and incubated for $24 \mathrm{hrs}$ at $37^{\circ} \mathrm{C}$ aerobically. After the incubation, the total CFU were calculated and tabulated.

Figure 1: Plate showing nil growth by decontamination with $2 \%$ glutaraldehyde solution

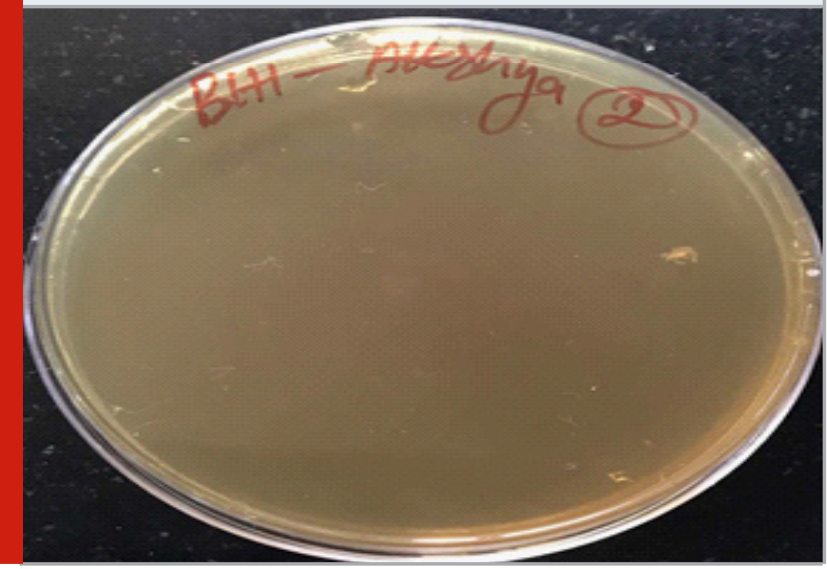

RESULTS AND DISCUSSION

The obtained results for this study are shown in table 1 and in figure 1, 2 and 3. Thus the tabulated results (Table 1 ) shows the comparison of $6 \%$ sodium 
hypochlorite and 2\% glutaraldehyde in decontamination of surgical instruments. Bar graph included in this study (Figure 3) depicts the number of bacterial colonies found in comparing 6\% sodium hypochlorite with $2 \%$ glutaraldehyde in decontamination of surgical instruments.

Figure 2: Plate showing growth with decontamination of $6 \%$ sodium hypochlorite for solution in an agar plate

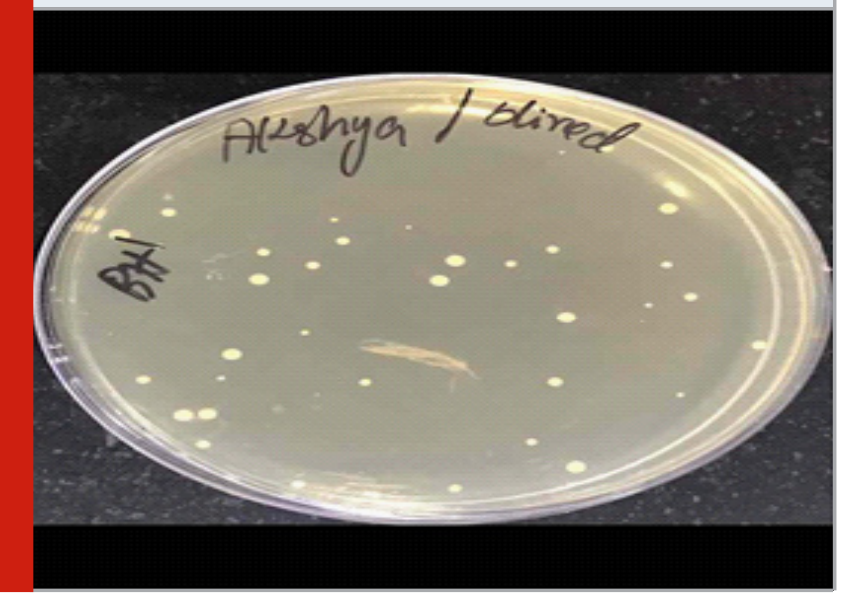

Table 1. Shows the comparison of decontamination of surgical instruments with $6 \%$ sodium hypochlorite and $2 \%$ glutaraldehyde in $\mathrm{CFU} / \mathrm{ml}$. It is clear that the amount of bacterial colonies reduced more in $2 \%$ glutaraldehyde than $6 \%$ sodium hypochlorite solution.

\begin{tabular}{|l|c|c|}
\hline Sample & $\begin{array}{c}\text { 6\% Sodium } \\
\text { hypochlorite }\end{array}$ & $\begin{array}{c}2 \% \\
\text { Glutaraldehyde }\end{array}$ \\
\hline 1 & 3800 & 100 \\
\hline 2 & 100 & 0 \\
\hline 3 & 850 & 100 \\
\hline 4 & 400 & 100 \\
\hline 5 & 200 & 100 \\
\hline 6 & 350 & 200 \\
\hline 7 & 850 & 0 \\
\hline 8 & 2400 & 200 \\
\hline 9 & 400 & 300 \\
\hline 10 & 350 & 100 \\
\hline & Mean : 970 & Mean $: 150$ \\
\hline
\end{tabular}

Previous study concluded that among the disinfectants $0.5 \%$ Sodium hypochlorite and $2 \%$ glutaraldehyde are considered as effective spray disinfectant on irreversible hydrocolloid impression material. It is suggested that they are effective against gram positive and gram negative organisms (Dandakery et al., 2003). Efficacy of cidex plus $3.2 \%$ glutaraldehyde solution has been evaluated for the disinfection of fiberoptic endoscopes. It is said that it has greater antimicrobial property against bacterial spores, vegetative organisms, mycobacterium and fungi. It is concluded that it is more effective that $2 \%$ glutaraldehyde (Akamatsu et al., 1997). 2\% glutaraldehyde has greater efficacy as disinfectant in arthroscopy and its procedures. It has been estimated that 2 percent glutaraldehyde is effective as a disinfecting agent when used for twenty minutes of cold soaking of metal and glass arthroscopic surgical instruments (Johnson et al., 1982). Thus it has been concluded that $2 \%$ glutaraldehyde is more effective in decontamination of surgical instruments than $6 \%$ sodium hypochlorite.

Figure 3: Bar Graph showing the comparison of mean CFU of $6 \%$ sodium hypochlorite and $2 \%$ glutaraldehyde solution in decontamination of surgical instruments. $\mathrm{X}$ axis represents the mean CFU of the samples collected and $\mathrm{Y}$ axis represents the bacterial count. It is concluded that $6 \%$ of sodium hypochlorite (Blue) shows more bacterial count than $2 \%$ glutaraldehyde (Orange) solution in decontamination of surgical instruments.

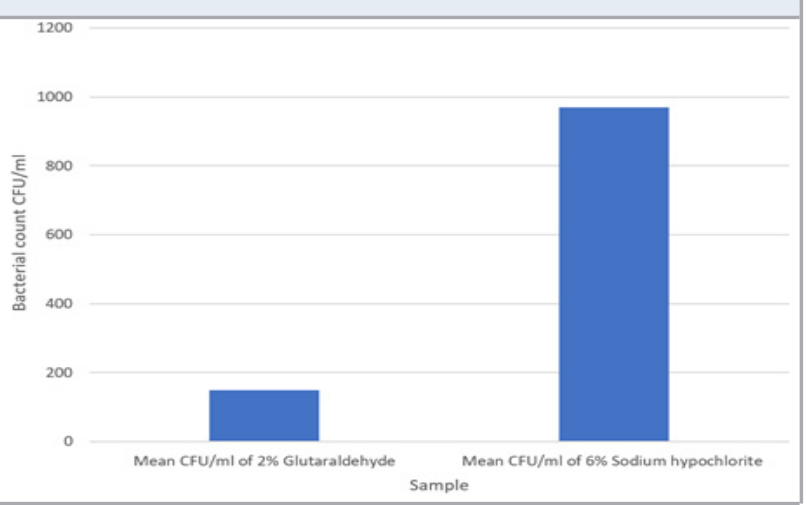

\section{CONCLUSION}

Surgical instruments are used in dental clinics, hospitals etc. Most of the recorded concentration shows that there is a vast difference seen between 6\% sodium hypochlorite and $2 \%$ glutaraldehyde solution. It is seen that $2 \%$ glutaraldehyde shows best results compared to that of $6 \%$ sodium hypochlorite solution in decontamination of soiled surgical instruments. Though both the chemical solutions are universally accepted for decontamination of soiled surgical instruments.

\section{ACKNOWLEDGEMENTS}

We thank saveetha dental college for the support to conduct the research study.

\section{Conflict of Interest: Nil}

\section{REFERENCES}

Akamatsu, T. et al. (1997) 'Evaluation of the efficacy of a 3 2\% glutaraldehyde product for disinfection of fibreoptic endoscopes with an automatic machine', The Journal of hospital infection. Elsevier, 35(1), pp. 47-57. doi: 10.1016/S0195-6701(97)90167-5.

Angelillo, I. F. et al. (1998) 'Evaluation of the efficacy of glutaraldehyde and peroxygen for disinfection of dental instruments', Letters in applied microbiology. 
Wiley Online Library, 27(5), pp. 292-296. Available at: https://www.ncbi.nlm.nih.gov/pubmed/9830148.

Ashwin, K. S. and Muralidharan, N. P. (2015) 'Vancomycin-resistant enterococcus (VRE) vs Methicillin-resistant Staphylococcus Aureus (MRSA)', Indian journal of medical microbiology. search.proquest. com, 33 Suppl, pp. 166-167. doi: 10.4103/02550857.150976.

Cardoso, C. L. et al. (1999) 'Rapid decontamination of gutta-percha cones with sodium hypochlorite', Journal of endodontia. Elsevier, 25(7), pp. 498-501. doi: 10.1016/ S0099-2399(99)80290-8.

Corcoran, G. D., Holton, J. and Ridgway, G. L. (1994) 'Endoscope decontamination: a comparison of the Wolf 35100 and DSD-91 systems', The Journal of hospital infection. Elsevier, 27(4), pp. 307-315. doi: 10.1016/0195-6701(94)90118-x.

Dandakery, S. et al. (2003) 'The effect of 0.5\% sodium hypochlorite and $2 \%$ glutaraldehyde spray disinfectants on irreversible hydrocolloid impression material', Indian journal of dental research: official publication of Indian Society for Dental Research. ncbi.nlm.nih.gov, 14(4), pp. 187-193. Available at: https://www.ncbi.nlm.nih.gov/ pubmed/15328983.

Fukuzaki, S. (2006) 'Mechanisms of actions of sodium hypochlorite in cleaning and disinfection processes', Biocontrol science. jstage.jst.go.jp, 11(4), pp. 147-157. doi: 10.4265/bio.11.147.

Johnson, L. L. et al. (1982) 'Two per cent glutaraldehyde: a disinfectant in arthroscopy and arthroscopic surgery', The Journal of bone and joint surgery. American volume. Citeseer, 64(2), pp. 237-239. Available at: https://www.ncbi.nlm.nih.gov/pubmed/6799519.

Mekonnen, D. et al. (2015) 'Evaluation of the efficacy of bleach routinely used in health facilities against Mycobacterium tuberculosis isolates in Ethiopia', The Pan African medical journal. ajol.info, 21, p. 317. doi: 10.11604/pamj.2015.21.317.5456.

Ozalp, N., Okte, Z. and Ozcelik, B. (2006) 'The rapid sterilization of gutta-percha cones with sodium hypochlorite and glutaraldehyde', Journal of endodontia. Elsevier, 32(12), pp. 1202-1204. doi: 10.1016/j. joen.2006.08.009.

Paiva, S. S. M. et al. (2013) 'Clinical antimicrobial efficacy of $\mathrm{N}$ i $\mathrm{T}$ i rotary instrumentation with $\mathrm{N}$ a OC 1 irrigation, final rinse with chlorhexidine and interappointment medication: a molecular study', International endodontic journal. Wiley Online Library, 46(3), pp. 225-233. Available at: https://onlinelibrary. wiley.com/doi/abs/10.1111/j.1365-2591.2012.02111.x.

Pereira, S. S. P. et al. (2015) 'Disinfection with sodium hypochlorite in hospital environmental surfaces in the reduction of contamination and infection prevention: a systematic review', Revista da Escola de Enfermagem da U S P. Revista da Escola de Enfermagem da USP, 49(4), pp. 0681-0688. doi: 10.1590/S0080623420150000400020.

Rani, L. and Others (2016) 'Sterilization protocols in dentistry-A review', Research journal of pharmaceutical, biological and chemical sciences. Journal of Pharmaceutical Sciences and Research, 8(6), p. 558. Available at: http://search.proquest.com/ openview/9cf7f1ff907efe2bc28d950e7520b6c4/1?pqorigsite $=$ gscholarEtcbl $=54977$.

Renuka, S. and Muralidharan, N. P. (2017) 'Comparison in benefits of herbal mouthwashes with chlorhexidine mouthwash: A review', Asian J Pharm Clin Res.

Rutala, W. A. and Weber, D. J. (2008) 'Guideline for disinfection and sterilization in healthcare facilities, 2008'. stacks.cdc.gov. Available at: https://stacks.cdc. gov/view/cdc/47378.

Sanchez, E. and Macdonald, G. (1995) 'Decontaminating dental instruments: testing the effectiveness of selected methods', Journal of the American Dental Association . Elsevier, 126(3), pp. 359-62, 364, 366 passim. doi: 10.14219/jada.archive.1995.0181.

Shahana, R. Y. and Muralidharan, N. P. (2016) 'Efficacy of mouth rinse in maintaining oral health of patients attending orthodontic clinics', Research Journal of Pharmacy and Technology. A \& V Publications, 9(11), pp. 1991-1993. Available at: http://www.indianjournals. com/ijor.aspx?target=ijor:rjpt\&tvolume=9Ctissue=11Ctar ticle $=035$.

Vianna, M. E. et al. (2004) 'In vitro evaluation of the antimicrobial activity of chlorhexidine and sodium hypochlorite', Oral surgery, oral medicine, oral pathology, oral radiology, and endodontics. Elsevier, 97(1), pp. 79-84. doi: 10.1016/s1079-2104(03)00360-3. 